\title{
BLETL - A PYTHON PACKAGE FOR INTEGRATING MICROBIOREACTORS IN THE DESIGN-BUILD-TEST-LEARN CYCLE
}

\author{
Michael Osthege ${ }^{+, 1,2}$, Niklas Tenhaef ${ }^{+, 1}$, \\ Rebecca Zyla ${ }^{1}$, Carolin Müller ${ }^{1,2}$, Johannes Hemmerich ${ }^{1}$, \\ Wolfgang Wiechert ${ }^{1,3}$, Stephan Noack ${ }^{1}$, Marco Oldiges ${ }^{*, 1,2}$ \\ ${ }^{+}$Contributed equally \\ ${ }^{1}$ Forschungszentrum Jülich GmbH, 52428 Jülich, Germany \\ ${ }^{2}$ RWTH Aachen University, Institute of Biotechnology, 52062 Aachen, Germany \\ ${ }^{3}$ RWTH Aachen University, Computational Systems Biotechnology (AVT.CSB), 52062 Aachen, Germany \\ ${ }^{*}$ Corresponding author
}

August 23, 2021

Keywords BioLector $\cdot$ feature extraction $\cdot$ growth rate $\cdot$ microbial phenotyping $\cdot$ uncertainty quantification

\section{Acronyms}

bletl BioLector Extract, Transform, Load.

BS backscatter.

DBTL Design - Build - Test - Learn.

DO Dissolved Oxygen.

FAIR Findability, Accessibility, Interoperability, Reusability.

IPTG Isopropyl- $\beta$-D-thiogalactopyranosid.

MAP maximum a-posteriori.

MBR microbioreactor.

MCMC Markov-chain Monte Carlo.

NUTS No-U-Turn Sampler.

PCA Principal Component Analysis.

t-SNE t-distributed Stochastic Neighbor Embedding. 


\section{Practical Application}

The bletl package can be used to analyze microbioreactor datasets in both data analysis and autonomous experimentation workflows. Using the example of BioLector datasets, we show that loading such datasets into commonly used data structures with one line of Python code is a significant improvement over spreadsheet or hand-crafted scripting approaches. On top of established standard data structures, practitioners may continue with their favorite data analysis routines, or make use of the additional analysis functions that we specifically tailored to the analysis of microbioreactor time series.

Particularly our function to fit cross-validated smoothing splines can be used for on-line signals from any microbioreactor system and has the potential to improve robustness and objectivity of many data analyses. Likewise, our random walk based $\vec{\mu}_{t}$ method for inferring growth rates under uncertainty, but also the time-series feature extraction may be applied to on-line data from other cultivation systems as well.

\section{Abstract}

Microbioreactor (MBR) devices have emerged as powerful cultivation tools for tasks of microbial phenotyping and bioprocess characterization and provide a wealth of online process data in a highly parallelized manner. Such datasets are difficult to interpret in short time by manual workflows. In this study, we present the Python package bletl and show how it enables robust data analyses and the application of machine learning techniques without tedious data parsing and preprocessing. bletl reads raw result files from BioLector I, II and Pro devices to make all the contained information available to Python-based data analysis workflows. Together with standard tooling from the Python scientific computing ecosystem, interactive visualizations and spline-based derivative calculations can be performed. Additionally, we present a new method for unbiased quantification of time-variable specific growth rate $\vec{\mu}_{t}$ based on a novel method of unsupervised switchpoint detection with Student-t distributed random walks. With an adequate calibration model, this method enables practitioners to quantify time-variable growth rate with Bayesian uncertainty quantification and automatically detect switch-points that indicate relevant metabolic changes. Finally, we show how time series feature extraction enables the application of machine learning methods to MBR data, resulting in unsupervised phenotype characterization. As an example, t-distributed Stochastic Neighbor Embedding (t-SNE) is performed to visualize datasets comprising a variety of growth/DO/pH phenotypes. 


\section{Introduction}

The development of innovative bioprocesses is nowadays often carried out in a Design - Build - Test - Learn (DBTL) cycle [1], where fast iterations of this cycle are desired to shorten development times and therefore safe costs. This acceleration can be enabled by modern genetic engineering tools, lab automation and standardized data analysis pipelines. One aspect in the "Test" part of the DBTL cycle of a bioprocess is the cultivation of the microorganisms to be tested. This is often performed in microbioreactor systems, since they provide a good balance between adequate throughput and scalability of the results to laboratory scale bioreactors as the gold standard [2][3].

A typical microbioreactor system will provide transient monitoring of biomass formation, dissolved oxygen, $\mathrm{pH}$, and fluorescence. Usually, the researcher has access to additional environmental data such as temperature, shaking or stirrer frequencies, humidity, and gas atmosphere. Analyzing this heterogeneous, multi-dimensional data in a quick and thorough manner can be challenging, especially since vendor software often covers only a limited amount of use cases.

From our experience, most researchers try to alleviate such problems by employing more-or-less sophisticated spreadsheets, available with various software solutions. While presenting an easy way for simple calculations and visualizations, extensive analysis of the data quickly results in hardly maintainable documents, which are impossible for colleagues to comprehend, error-prone and easy to break. Most importantly, such multi-step manual data transformations do not comply with the FAIR data principles, whereas our bletl package directly addresses the accessibility aspect and creates incentives to, for example, retain the original data.

Automated data analysis pipelines solve this problem by removing the repetitive and error-prone manual workflows in favor of standardized workflows defined in code. Such workflows offer many advantages, if done correctly: a) data processing is clearly understandable and documented, b) every step is carried out for every input data file in the same way, guaranteeing the integrity and reproducibility of the results, c) data processing can be autonomously started after data generation and d) such a pipeline can be run on remote systems, which is especially useful for computational demanding calculations. Such data analysis pipelines are routinely used, e. g., for sequencing data [4], but seldom used for microbioreactor data. One example is the automated calculation of growth rates using MATLAB [5].

Additionally, automated data analysis opens up possibilities for at-line analysis and subsequent intervention during an experiment: Cruz Bournazou et al. report a framework for online experimental redesign using a data pipeline implemented in MATLAB [6]. Jansen et al. build a Python-based process control system to control pH and enzyme addition to a microbioreactor system using a liquid handling robot [7]. While those examples can automatically parse and analyze microbioreactor data, they are tailored to a specific use case and hence not universally applicable. 
In this study, we introduce bletl as an open-source Python package for standalone and at-line parsing and analysis of microbioreactor data. The name bletl is inspired by the fact that it simplifies the implementation of extract, transform, load data processing workflows specifically for BioLector datasets. It is capable of parsing raw data without involving vendor software, making necessary calibrations for fluorescent-based measurement of $\mathrm{pH}$ and dissolved oxygen, and presenting all measurement, environmental and meta data in the easily accessible, DataFrame format from the popular pandas library [8,9]. Currently, bletl is designed to parse data from the devices BioLector I, II, and Pro manufactured by Beckman Coulter Life Sciences, but its general design and methods can be applied also for other devices. Its analysis submodules build on top of bletl 's standardized data structures and provide the user with sophisticated methods for microbioreactor data analysis, such as spline approximation with cross validation for data smoothing, growth rate analysis using Bayesian modeling techniques as well as time series feature extraction. In addition to an extensive documentation and automated software tests, we provide application examples using relevant experimental data. With bletl, scientists using microbioreactors have a powerful tool to make their data analysis less cumbersome and error-prone, while they can directly benefit from state-of-the-art machine learning techniques.

\section{Materials and Methods}

\subsection{Core package}

The bletl package includes the data structures that are common to datasets originating from BioLector I, II and Pro microbioreactors. Parsing of raw data is deferred to parsers that may implement logic that is specific to a certain BioLector model or file type.

\subsubsection{Parsing and data structures}

Parsing of raw data typically begins with a call to the bletl.parse function, which first determines the file type from its content. The parsing procedure does not only ingest the data into accessible Python data structures, but also takes care of re-naming tabular data columns to a standardized naming scheme and type-casting values to integer, float or string types. After the BioLector model and file type version are identified, a matching parser is selected, thereby enabling a plug-in system for specialized parsers for different file type versions or the new BioLector XT device. The parsing logic is highly dependent on the BioLector model, but generally follows the pattern of first separating the file header of metadata from the table of measurement data. Logical blocks of information, such as the table of filtersets are then parsed into pandas. DataFrame objects. These tabular data structures are collected as attributes on a bletl. BLData object which is returned to the user. The BLData class is a Python dictionary data type with additional properties and methods. Via its properties, the user may access various DataFrame tables of relevant metadata, including the aforementioned tables of filtersets, comments or environment parameters such as chamber temperature or humidity. 
Key-value pairs in the BLData dictionary are the names of filtersets and corresponding FilterTimeSeries objects. This second important type from the bletl package hosts all measurements that were obtained with the same filterset. Like the BLData class it provides additional methods such as FilterTimeSeries.get_timeseries for easy access of time/value vectors.

\subsection{Analysis methods}

In submodules of the bletl package, various functions are provided to facilitate higher-throughput and automated data analysis from bioprocess timeseries data.

One often used feature is the find_do_peak function that implements a Dissolved Oxygen (DO)-peak detection heuristic similar to the one found in the m2p-labs RoboLector software. The DO-peak detection algorithm finds a cycle number corresponding to a DO rise, constrained by user-provided threshold and delay parameters.

Additional, more elaborate analysis functions were implemented to allow for advanced data analysis or experimental control.

\subsubsection{Spline approximations}

To accommodate for the measurement noise in on-line measured time series, various smoothing procedures may be applied to the raw signal. A popular choice for interpolation are spline functions, specifically smoothing splines that can reproduce reasonable interpolations without strong assumptions about the underlying relationship. With bletl.get_crossvalidated_smoothing_spline we implemented a convenience function for fitting smoothing splines using either scipy or csaps $[10,11]$ for the underlying implementation. Both smoothing spline implementations require a hyperparameter that influences the amount of smoothing. Because the choice of the smoothing hyperparameter strongly influences the final result we automatically apply stratified k-fold cross-validation for determining its optimal value. The implementation can be found in the code repository of the bletl project [12].

\subsubsection{Growth rate analysis}

A "calibration-free" approach to calculate time-variable specific growth rate $\mu(t)$ (1) relies on the previously introduced spline approximations, combined with the popular assumption of a linear backscatter $Y_{B S}$ vs. biomass $X$ relationship (2).

$$
\begin{gathered}
\mu(t)=\frac{d X}{d t} \cdot \frac{1}{X(t)}=\frac{\dot{X}(t)}{X(t)} \\
Y_{B S}(t)=a \cdot X(t)+b \\
\Leftrightarrow \quad X(t)=\frac{Y_{B S}(t)-b}{a}
\end{gathered}
$$


Substituting the biomass $X(t)$ in (1), the slope parameter $a$ cancels out such that only a "blank" $b$ and the measured backscatter $Y_{B S}(t)$ are needed for a specific growth rate calculation (3).

$$
\begin{aligned}
& \Leftrightarrow \mu(t)=\frac{1}{a} \cdot \frac{d\left(Y_{B S}(t)-b\right)}{d t} \cdot \frac{a}{Y_{B S}(t)-b} \\
& \Leftrightarrow \mu(t)=\frac{1}{Y_{B S}(t)-b} \cdot \frac{d\left(Y_{B S}(t)-b\right)}{d t}
\end{aligned}
$$

Finally, the backscatter curve $Y_{B S}(t)$ can be approximated by a smoothing spline $S_{\mathrm{YS} \text {,blanked }}(t)$ to obtain a differentiable function (4).

$$
\mu(t)=\frac{\dot{S}_{\mathrm{YS}, \text { blanked }}(t)}{S_{\mathrm{YS}, \text { blanked }}(t)}
$$

An alternative approach is to construct a generative model of the biomass growth. In essence, the time series of observations is modeled as a deterministic function of an initial biomass concentration $X_{0}$ and a vector of specific growth rates $\vec{\mu}_{t}$ at all time points where observations were made. The structure of this model assumes exponential growth between the time steps, which is a robust assumption for high-frequency time series such as the ones obtained from BioLector processes.

$$
\vec{\mu}_{t}=X_{0} \cdot e^{\operatorname{cumsum}\left(\vec{\mu}_{t} \odot \overrightarrow{\Delta t}\right)}
$$

where

$$
\begin{aligned}
\operatorname{cumsum}(\vec{x}) & :=\sum_{i=0}^{T} x_{i} \\
\overrightarrow{\Delta t} & =\text { differences between time points }
\end{aligned}
$$

The convenience function bletl.growth.fit_mu_t creates the generative $\vec{\mu}_{t}$ model (5) from user-provided vectors of observation time points $\vec{t}$ and backscatter values $\vec{y}$. To contrast it from the smooth, continuous $\mu(t)$ from (4) we use the $\vec{\mu}_{t}$ notation to underline that the approach discretizes the growth rate into a step function. The model is built with the probabilistic programming language PyMC3 $[13,14]$ and an optimal parameter set, the maximum a-posteriori (MAP) estimate, is found automatically. Additionally, the user may decide to perform Markov-chain Monte Carlo (MCMC) sampling using advanced sampling algorithms such as No-U-Turn Sampler (NUTS) from the PyMC3 [13] package to infer probability distributions for the model parameters $X_{0}$ and $\vec{\mu}_{t}$.

In the generative $\mu_{t}$ model, the vector of growth rates is modeled with either a Gaussian or Student-t distributed random walk (Figure 1). This does not only result in a smoothing of the growth rate vector, but enables additional flexibility with respect to switchpoints in the growth rate. A drift_scale parameter must be given to configure the random walk with a realistic assumption of how much growth rate varies over time. Small drift_scale corresponds to the assumption that growth rate is rather stable, whereas large drift_scale allows the model to describe a more fluctuating growth rate distribution. Additionally, the user may provide previously known time points at which growth rate switches are 
expected. Examples of such switchpoints are the time of induction, occurrence of oxygen limitation or the time at which the carbon source is depleted. If $\vec{\mu}_{t}$ is described by a Student-t random walk, switchpoints can be detected automatically by inspecting the prior probabilities of the estimated growth rate in every segment (Figure 1). Our implementation automatically classifies elements of $\vec{\mu}$ as switchpoints as soon as their prior probability is $<1 \%$.

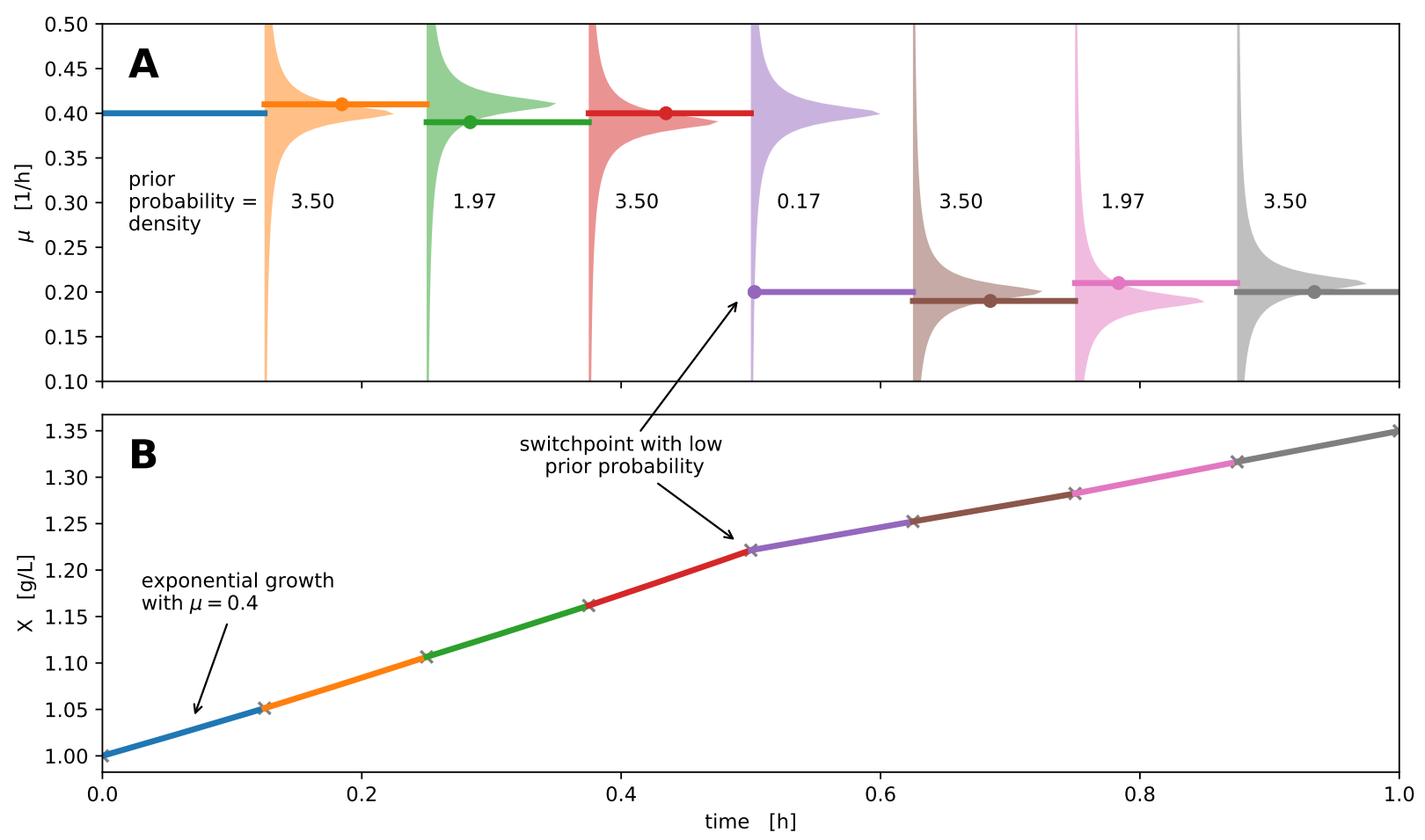

Figure 1: Switchpoint detection with a Student-t random walk

A microbial growth curve with fluctuating specific growth rate $\mu$ may be discretized into segments of exponential growth with constant growth rate (solid lines). Modeling such a sequence of growth rates with a random walk assigns prior probabilities to every value of $\mu \in \vec{\mu}$, centered on the values of previous iterations (A, colored areas). When fitting the model these prior probabilities "pull" subsequent values in $\vec{\mu}$ towards each other, leading to a smoothing and counteracting overfitting. Using a fat-tailed Student-t distribution for the random walk prior, the penalty for large jumps is less extreme compared to a Normal distribution, thereby allowing for jumps in the random walk (5th segment).

While there has been prior work on using random walks for outlier detection [15] we are not aware of any prior work using Student-t random walks for the unsupervised detection of switchpoints in time series data.

\subsubsection{Feature extraction}

The bletl.features submodule implements functions for the automated extraction of both biologically and statistically motivated time series features. An abstract Extractor class may be inherited to implement feature extraction of characteristic features such as DO peaks. Additionally, our TSFreshExtractor uses the open source Python package tsfresh [16] to extract hundreds of features from variable length time series automatically. Starting from a bletl.BLData object containing one or more FilterTimeSeries, the bletl.features.from_bldata function extracts features from multiple filtersets using a user-specified mapping of Extractor objects. Optionally, a dictionary of well-wise cycle numbers can be passed to truncate time series to the relevant cycles. The results are returned as a 
DataFrame for maximal compatibility with downstream analysis operations. For details on the implementation we refer to the code and documentation [12, 17].

Figure 2 shows how the function is applied to our demonstration data set. The resulting DataFrame comprised 2343 feature columns for each of the 48 wells in the input data. Feature columns with NaN, $\pm \infty$ entries or without variability in their values were dropped, resulting in 1282 features available for further analysis.

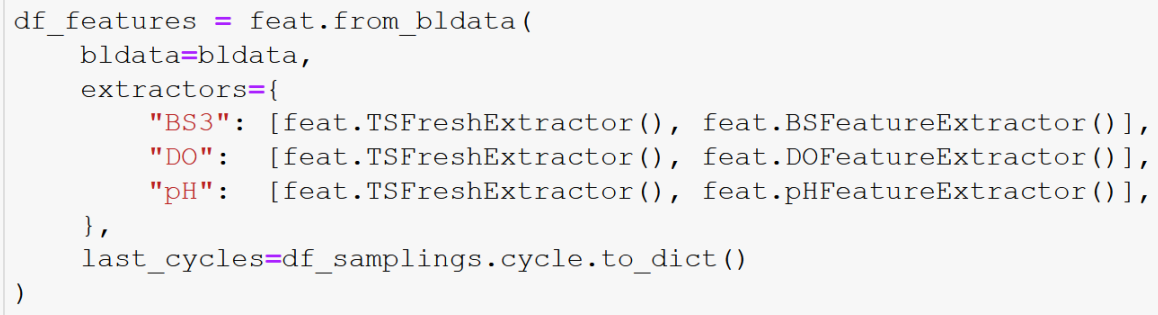

Figure 2: Code to run feature extraction from three filtersets

The name of each filterset is mapped to a list of Extractors that may include user-defined feature extraction implementations. The last_cycle keyword argument can be used to pass a mapping of well IDs to the last relevant cycle numbers. Extracted features are returned in the form of a pandas. DataFrame.

\subsubsection{Visualization by t-SNE}

Starting from features extracted with bletl. features we applied the t-SNE technique to find a two-dimensional embedding for the visualization of local structure in the dataset. The extracted time series features were cleaned such that features with NaN values, or without diversity were removed. After feature-cleaning, the t-SNE implementation from scikit-learn was applied with a perplexity setting of 10 and initialization by PCA.

\subsection{Media and cultivation conditions}

The dataset presented as an application example in this study was obtained in an automated cultivation workflow on the previously described microbial phenotyping platform. 48 cultures of Corynebacterium glutamicum ATCC 13032 harboring the pPBEx2[18]-based plasmid pCMEx8-NprE-Cutinase (sequence in section 4.3) were cultivated in CGXII medium (recipe as in [19]) with different carbon sources. Carbon sources were prepared as C-equimolar, random combinations of $8 \frac{g_{C}}{L}$ glucose, fructose, maltose, sucrose, gluconate, lactate, glutamate or myo-inositol. For every well except A01, where glucose was the sole carbon source, three different carbon sources were chosen at random. A total of $140 \mu \mathrm{L}$ of C-equimolar carbon source stock were added to each well. The $140 \mu \mathrm{L}$ were split into 7 parts of $20 \mu \mathrm{L}$ and such that at least one part was used for each selected carbon source and the remaining 4 parts were assigned randomly. The resulting media composition in terms of pipetted volume, and carbon mass per $\mu \mathrm{L}$ can be found in Section 4.1. $800 \mu \mathrm{L}$ CGXII medium were inoculated to an optical density at $600 \mathrm{~nm}$ of 0.2 . Cultures were grown in a MTP-48-BOH 1 FlowerPlate in a BioLector Pro (both Beckman Coulter Life Sciences, USA) at $1400 \mathrm{rpm}, 30^{\circ} \mathrm{C}$ and $\geq 85 \%$ humidity. Expression was induced autonomously with $10 \mu \mathrm{L}$ Isopropyl- $\beta$-D-thiogalactopyranosid (IPTG) (final concentration 
$100 \mu \mathrm{M}$ ) when cultures reached a backscatter value of 5.82, corresponding to approximately $4 \frac{g_{C D W}}{L}$. Culture from each well was harvested 4 hours after induction [20].

\section{Results and Discussion}

\subsection{Basic visualization workflow}

Every analysis begins with loading data into a structure that can be used for further analysis. In the case of a BioLector experiment, the data are multiple tables that hold information about filtersets, environment variables such as temperature or humidity, as well as the well-wise measurements. In most cases the result files already contain relevant meta information such as lot number or process temperature and parsing them with bletl comes down to a single line of Python (Figure 3).

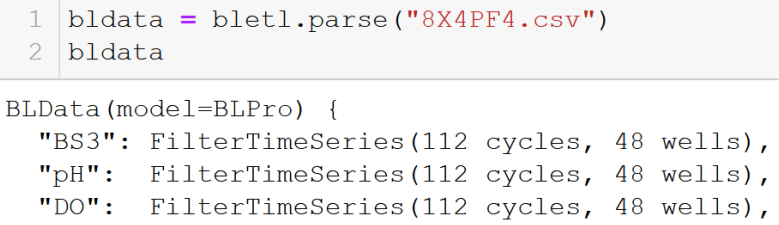

Figure 3: Parsing of a BioLector result file

The bletl . parse function automatically determines the file type (BioLector I, II or Pro) and applies calibration of optode measurements based on lot number and temperature from the file. Optionally, lot number and temperature, or calibration parameters may be passed to override the values from the file. The function can also process a list of result file paths and automatically concatenate them to a single BLData object.

The bletl. BLData type is a dictionary-like data structure into which results are loaded. It has additional properties through which process metadata and measurements that are not tied to individual wells can be obtained. Its text representation, which appears when the object is displayed in an interactive Jupyter notebook session shows the names of filtersets and the amount of data they contain (Figure 3).

Elements in the BLData object are the FilterTimeSeries, that contain the well-wise measurements. The simplest way to access the time series of a particular filterset and well is via the BLData.get_timeseries (well, filterset) or FilterTimeSeries.get_timeseries (well) methods. Optionally, a last_cycle number may be passed to retrieve only the data up to that specific cycle number. This is useful in situations where wells were sampled and might only be analyzed up to the sampling time point.

With the data structures provided by our bletl package, the data analysis workflow for a BioLector experiment is no different to any standard data analysis performed with Python. Figure 4 shows an example how a simple figure of process variables can be prepared using the popular matplotlib visualization library. Such a visualization of measurements, together with annotations of, for example, measurement pausing events can help to diagnose irregularities, or to provide a quick overview about the experiment.

In the dataset presented here, cultures were induced and sampled by a robotic liquid handler. Induction was triggered 


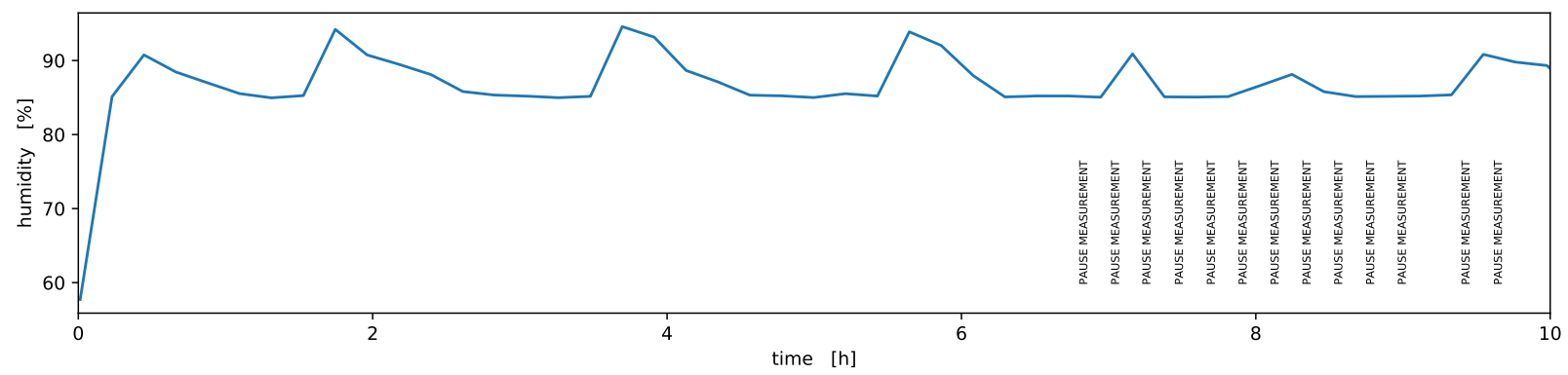

Figure 4: Visualization of process variables

This screenshot shows how a simple figure of humidity over time can be prepared alongside annotations of "pause measurement" events. In lines 4 and 5 the time and value vectors for humidity measurements are fetched from the environment pandas. DataFrame property of the previously loaded data object. The for-loop in lines 8-13 iterates over entries of another table comments that holds user- and system-comments of the BioLector process.

at-line based on latest backscatter observations and sampling was performed 4 hours after the induction events Section 2.3. The meta data of these induction and sampling events were logged into an XLSX file and loaded into a pandas. DataFrame for the analysis Table 1.

Table 1: Excerpt of sampling event $\log$

\begin{tabular}{llrrrl}
\hline & timestamp & time & cycle & volume & supernatant_well \\
well & & & & & \\
\hline A01 & $2020-07-21 \mathrm{~T} 08: 10: 04.721 Z$ & 13.076466 & 61 & -950 & H01 \\
A02 & $2020-07-21 \mathrm{~T} 07: 05: 04.299 \mathrm{Z}$ & 11.993016 & 56 & -950 & G01 \\
A03 & $2020-07-21 \mathrm{~T} 08: 10: 04.786 Z$ & 13.076485 & 61 & -950 & F01 \\
A04 & $2020-07-21 \mathrm{~T} 08: 10: 04.841 Z$ & 13.076500 & 61 & -950 & E01 \\
A05 & $2020-07-21 \mathrm{~T} 06: 26: 17.384 Z$ & 11.346651 & 53 & -950 & D01 \\
\hline
\end{tabular}

The meta information about induction and sampling events is important for the analysis, because backscatter, $\mathrm{pH}$ and DO observations made after a well was sacrifice-sampled must be truncated before analysis or visualization.

Most data analyses are driven by interactive exploration of the data. With bletl, or rather with a Python-based data analysis workflow in general, this is facilitated by interactive plots using helper functions from, for example, the ipywidgets library. Figure 5 shows the code and resulting interactive plot of measurement results from a BioLector dataset. In comparison to Figure 4 the example shown in Figure 5 is marginally more involved, but again relies on standard tooling from the Python ecosystem. In this case the ipywidgets library is used to wrap a plotting function and create interactive input elements for selecting the filterset and wells to show.

\subsection{Splines for time series smoothing and derivatives}

Optical on-line measurements as those performed by the BioLector are inevitably subject to measurement noise. While the measurement noise of DO and pH signals in the BioLector II/Pro system was greatly reduced compared to the BioLector I model, it still requires special attention in subsequent data analysis procedures. Particularly in automated at-line decision making such as triggered induction or sampling, measurement noise can cause problems 


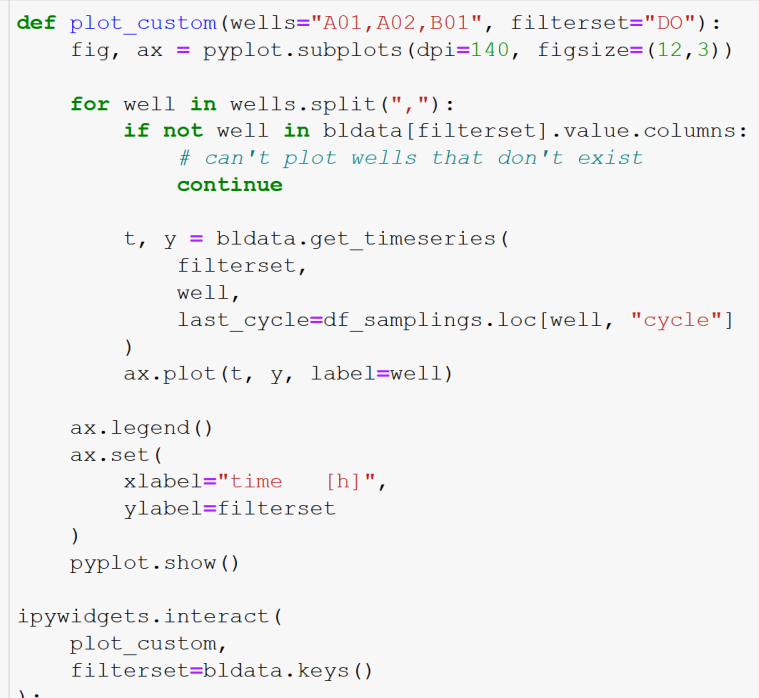

$$
\text { A }
$$

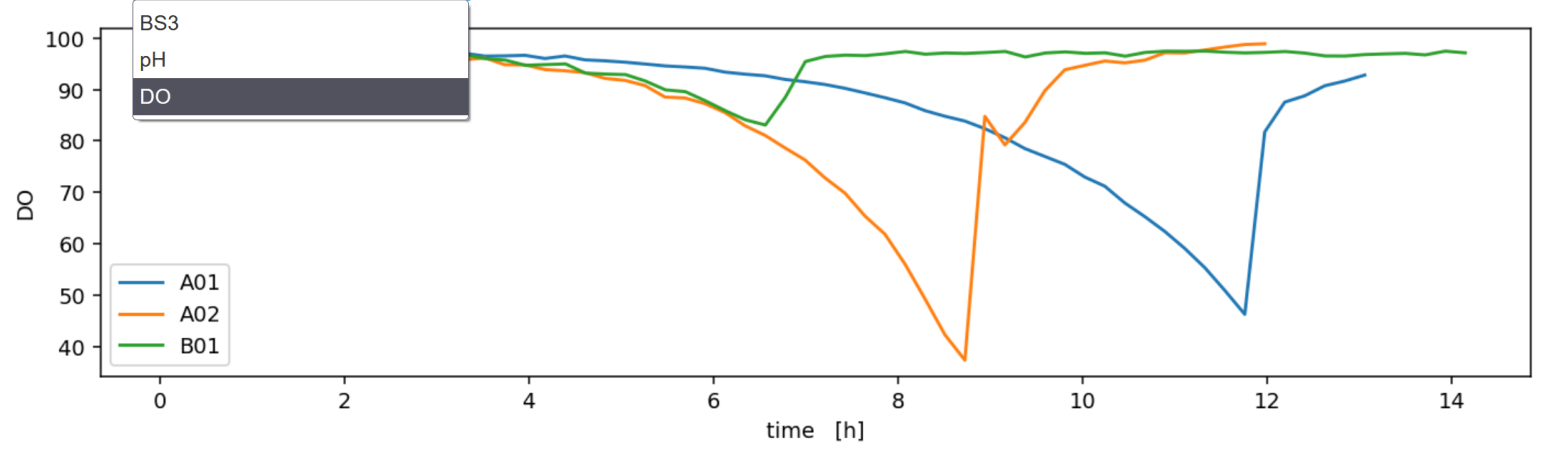

Figure 5: Interactive plot of well-wise measurements

A plot_custom function, defined in lines 1-17 takes a comma-separated text of well IDs and the name of a filterset as parameters for the visualization. In line 4 it iterates over the well IDs to create lines plots of the measurements, passing the number of the last relevant cycle from the event $\log$ (Table 1) to truncate the data. Line 21 passes the list of filtersets in the dataset (Figure 3) as options for the $\mathrm{fs}$ keyword-argument of the plotting function, thereby populating the dropdown menu.

with threshold-based heuristics. With noisy on-line signals, such as optode measurements in BioLector I datasets, smoothing splines can yield more accessible visualizations and allow for finer-grained comparisons. Furthermore, the slope of the signals may be used for more sophisticated analysis or decisions.

For at-line triggers based on such noisy process values, a smoothing of the signal can increase the reproducibility of detecting, for example, a pH threshold. At the same time, the slope of process values often gives more process insight compared to absolute values alone. For example, a dissolved oxygen tension of $60 \%$ alone is not very meaningful, but the observation of a strong positive slope tells the process engineer that the microbes might grow with reduced oxygen uptake rate. The calculation and visualization of $\mathrm{pH}$ and $\mathrm{DO}$ slopes is therefore an important tool for process data analysis.

Splines are a popular choice for both smoothing and derivative calculation, because they make few assumptions about the 
data and are available in most standard data analysis software. There are however multiple flavors of smoothing splines and they come with a smoothing parameter whose value has a considerable effect on the results. In bletl.splines we implemented a convenience function that automatically performs k-fold cross-validation on the smoothing parameter of either a UnivariateSpline cubic spline from scipy or a UnivariateCubicSmoothingSpline from csaps (cf. Section 2.2.1).
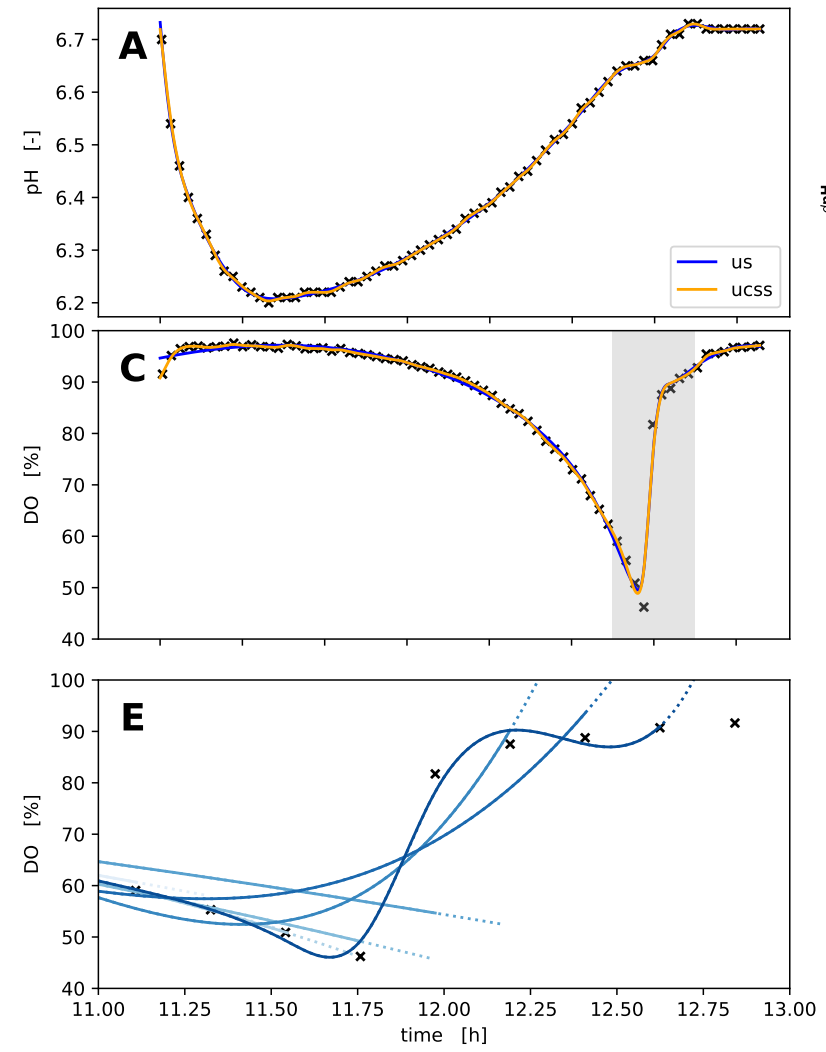
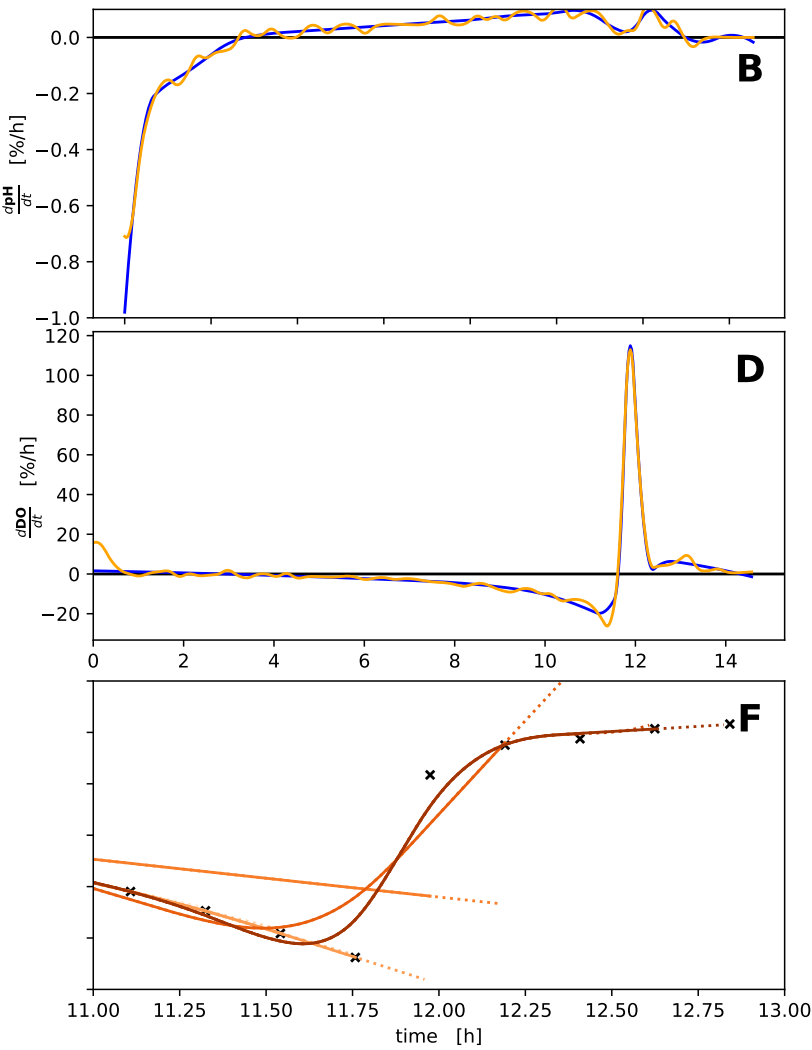

Figure 6: Splines fitted to $\mathrm{pH}$ and DO time series

Both spline methods "us" (blue) and "ucss" (orange) were applied to measurements of pH (A) and DO (C). The resulting reconstruction/interpolation is largely identical $(\mathbf{A}, \mathbf{C})$ with most notable differences between the methods at the start/end, as well as their derivatives $(\mathbf{B}, \mathbf{D})$, where the "ucss" method has considerably more wiggly derivatives. In the bottom row (E, F), the extrapolation (dotted lines) of splines fitted to data subsets of different length (solid lines) is straight for the "ucss" method, whereas the "us" method extrapolates with a curvature.

In Figure 6 the two spline methods were applied to $\mathrm{pH}$ and DO time series of well A01 from our demonstration dataset. Both smoothing spline methods find interpolations (solid lines) of the raw data that are almost indistinguishable. Their 1st derivative however reveals that the UnivariateCubicSmoothingSpline (ucss) from the csaps package is much more wiggly compared to the UnivariateSpline (us) from SciPy.

The bottom row shows a comparison of both methods in a simulated at-line situation where the DO time series grows point by point at the time of the characteristic substrate-depletion DO-peak. In this situation the "us" method produces strong alternating positive or negative slopes and curved extrapolation at the end of the curve. In contrast, the splines obtained with the "ucss" method extrapolate with an (almost) constant slope. Taking both scenarios into account, the 
choice between the "us" and "ucss" depends on the use case. As a rule of thumb, "us" is more suited when steady derivatives are desired, whereas the more stable extrapolation of the "ucss" splines should be preferred for at-line applications.

\subsection{Growth rate and timeseries analysis}

Most cultivations in microbioreactors such as the BioLector are conducted to extract key performance characteristics of the bioprocesses from the on-line measurements. One such performance indicator is the specific growth rate $\mu$. In applications where unlimited exponential growth is observed, a constant maximum specific growth rate $\mu_{\max }$ can be calculated by regression with an exponential function [5]. Many processes however do not fulfill this assumption and require a more detailed analysis with time-variable specific growth rate. Unlimited exponential growth may be terminated by nutrient limitation, or the characteristics of strain and cultivation media may lead to multiple growth phases. For example, overflow metabolism of E. coli growth on glucose can lead to an accumulation of acetic acid which is metabolized in a second growth phase. Accordingly, switchpoints in growth rate can indicate limitations, changes in metabolism or regulation.

From temporally highly resolved backscatter observations combined with a detailed biomass/backscatter correlation model, variable specific growth rate can be calculated using our bletl.growth.fit_mu_t function. This model describes the data in a generative fashion by first discretizing time into many segments of exponential growth, followed by simulating the biomass curve resulting from a growth rate that drifts over time. For this it assumes an initial biomass concentration $X_{0}$ and a vector of growth rates $\vec{\mu}$, calculates biomass concentrations deterministically and compares them to the observed backscatter using a calibration model built with the calibr8 package [19]. Parameters $X_{0}$ and $\vec{\mu}$ can be obtained through optimization or MCMC. In this analysis we specified a prior belief in $X_{0}$ centered around $0.25 \mathrm{~g} / \mathrm{L}$, corresponding to typical inoculation density for BioLector experiments. The prior for $\vec{\mu}$ is a random walk of either a Normal or Students- $t$ distribution, which pulls the neighboring entries in the growth rate vector closer to each other, resulting in a smooth drift of $\vec{\mu}_{t}$ (Section 2.2.2). While this method makes few assumptions about the underlying process and therefore can be applied to many datasets, practitioners wanting to encode process knowledge should also consider differential-equation based modeling approaches for which Python packages such as pyFOOMB or murefi can be applied [19, 21].

To benchmark the objectivity of the method, we generated a synthetic dataset from a vector of growth rates (Figure $7 \mathbf{A}, \mathbf{B}$ ). The comparison of the inference result with the ground truth (Figure 7) shows that with the correct calibration model it yields unbiased estimates of the underlying growth rate. Figure 7 also shows that the drift_scale parameter can be tuned to reflect an assumption about the stability of growth rate in the model. Low drift_scale constrains the model towards stable exponential growth and correspondingly narrow uncertainties (Figure 7, C, D). Large drift_scale on the other hand encodes the prior belief that growth rate is unstable, leading the model to infer rather unstable growth rates with much higher uncertainty (Figure 7, E, F). 
bioRxiv preprint doi: https://doi.org/10.1101/2021.08.24.457462; this version posted August 25, 2021. The copyright holder for this preprint (which was not certified by peer review) is the author/funder, who has granted bioRxiv a license to display the preprint in perpetuity. It is made available under aCC-BY 4.0 International license.

bletl - A Python package for integrating microbioreactors in the Design-Build-Test-Learn cycle
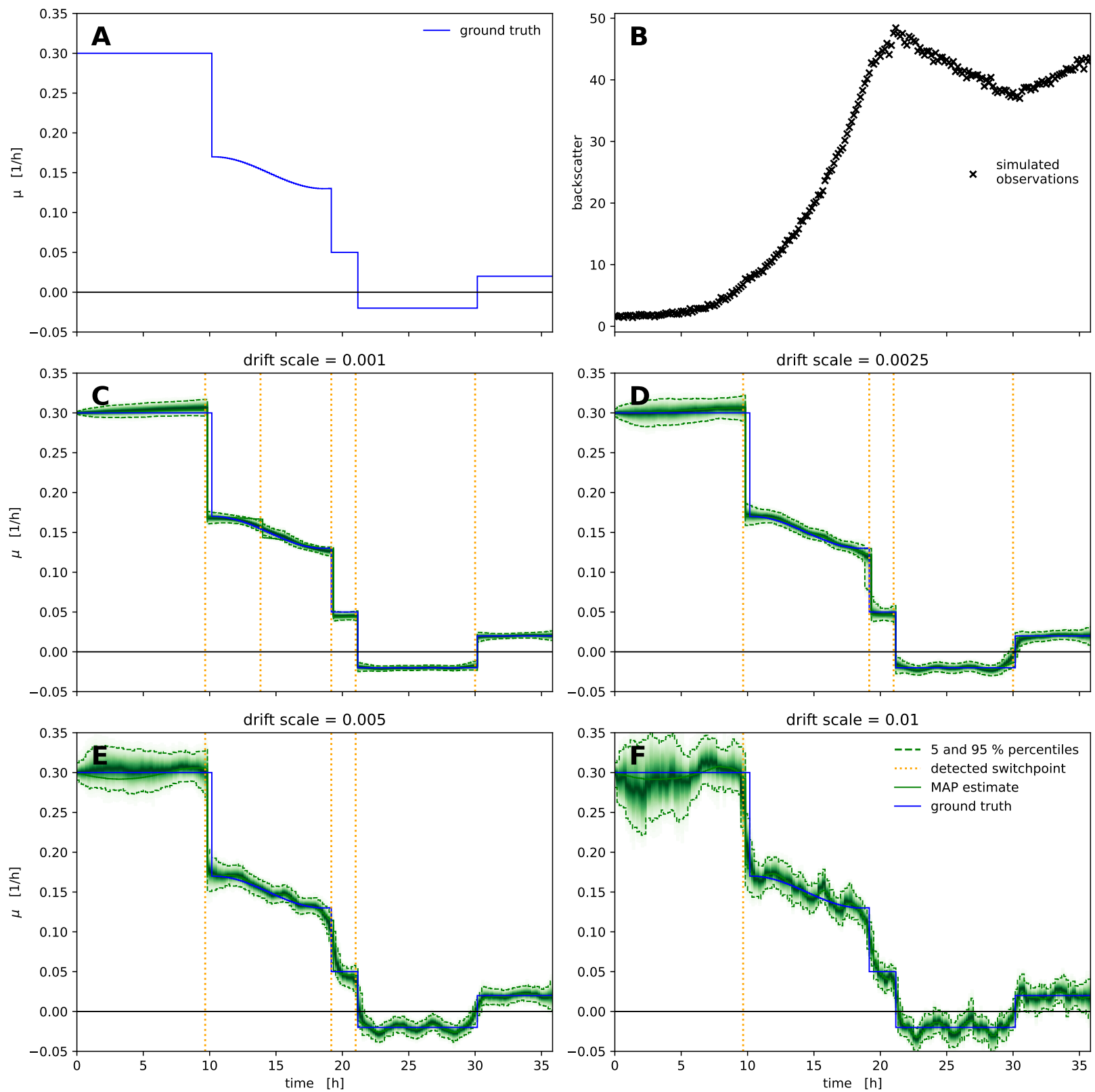

Figure 7: Inference of growth rate from a synthetic dataset

A vector of growth rates (A) exhibiting switchpoints and a smooth fluctuation was used to simulate biomass concentrations (not shown) and corresponding backscatter observations (B). The magnitude of the drift_scale parameter (scale of the Students-t distribution in the random walk) effects stability, switchpoint detection and uncertainty (C-F), but in all cases the model fit is unbiased compared to the ground truth. Small drift_scale settings constrain the model to stable growth rates, which are inferred with little uncertainty $(\mathbf{C}, \mathbf{D})$. Large drift_scale allows for larger variance in the growth rate, leading to more uncertainty and fewer automatically detected switchpoints $(\mathbf{E}, \mathbf{F})$. The green density bands visualize the posterior probability density, with dashed lines marking the 5 and $95 \%$ percentiles.

In Figure 8 we applied our generative $\vec{\mu}_{t}$ method to data from well F02 of the example dataset. The carbon source composition in this well were 3 parts fructose, 3 parts gluconate and 1 part lactate, causing a change in growth phase at around $9.35 \mathrm{~h}$. The orange line shows the maximum a-posteriori estimate of $\vec{\mu}_{t}$, obtained by optimization. Automatically detected growth rate switchpoints are shown as dashed lines. The green density visualizes the percentiles 

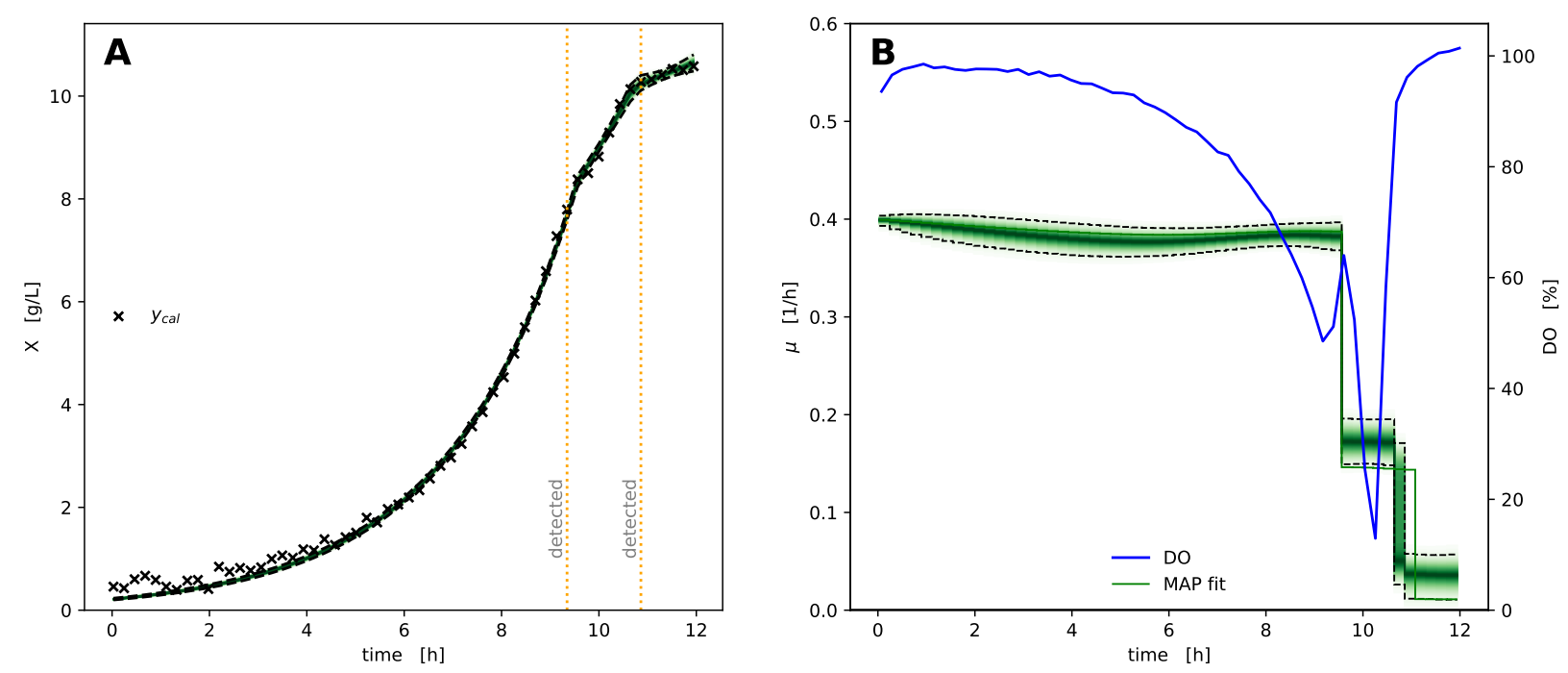

Figure 8: Model prediction of variable growth rate Biomass concentrations inferred from backscatter observations (A) are well explained by the drift of specific growth rate over time (B). At two timesteps the specific growth rate changed significantly, which resulted in the automatic detection of switchpoints. These switchpoints in $\vec{\mu}_{t}$ at 9.35 and $10.65 \mathrm{~h}$ coincide with changes in the Dissolved Oxygen (DO), indicating a change in cell metabolism. The green density bands visualize the posterior probability density, with dashed lines marking the 5 and $95 \%$ percentiles.

of the posterior probability distribution of the biomass concentration (left) and growth rate (right). The MAP estimate (orange line),is largely in agreement with the full posterior probability distribution obtained by MCMC. This similarity of MAP and the full posterior distribution is not always the case in Bayesian data analysis, but since the computational runtime to obtain the MAP estimate (seconds) is around 100x lower compared to the runtime of a full MCMC parameter estimation (minutes), it is often the first step when analyzing a new dataset.

The comparison of growth rate over time (right, orange/green) with dissolved oxygen tension (blue) shows that both detected switchpoints in the growth rate fall together with severe changes in the dissolved oxygen concentration. The first switch from $>0.4 \frac{1}{h}$ to $\approx 0.2 \frac{1}{h}$ coincides with a temporary increase in DO, whereas the second switch from $\approx 0.2 \frac{1}{h}$ to $\approx 0.05 \frac{1}{h}$ falls together with the final rise in oxygen concentration.

One key aspect of growth rate calculation are the assumptions made about the biomass/backscatter relationship. The aforementioned $\vec{\mu}_{t}$ method relies on a calibration model of backscatter vs. biomass concentration to simultaneously describe the relationship and measurement noise with a non-linear calibration model. This raises the question to what extend growth rate may be quantified with less sophisticated calibrations.

In Figure 9 we compare the results of a "calibration-free" $\mu(t)$ spline approach (Section 2.2.2) with the $\vec{\mu}_{t}$ method using linear or logistic calibration models. Note that the "calibration-free" approach also makes the assumption of a linear relationship between biomass concentration and backscatter observations, just without specifying the slope that cancels out in the growth rate calculation (Section 2.2.2).

Compared to the alternatives, the growth rate curve resulting from the spline method exhibits strong oscillatory artefacts at the beginning of the curve, where the biomass concentration is low. The blue density shows the results of the 
generative $\vec{\mu}_{t}$ method combined with a linear biomass/backscatter calibration that uses calibration data up to $6 \mathrm{~g} / \mathrm{L}$ and fixes the intercept to a blank value. This model can still detect the switchpoints, but is biased towards considerably higher growth rates (blue). In contrast, a linear calibration with 6-30 g/L that does not fix the intercept parameter to a blank value leads to a strong under-estimation of the growth rate, largely explained by the lack of fit error of the calibration model (Figure S1). For detailed guidance on the construction and diagnosis of calibration models we refer to [19].

The strength of non-linearities in the biomass/backscatter relationship may depend on the BioLector model and device at hand, but one must conclude that a realistic, unbiased biomass/backscatter calibration is indispensable when quantitative estimates of specific growth rates are desired.

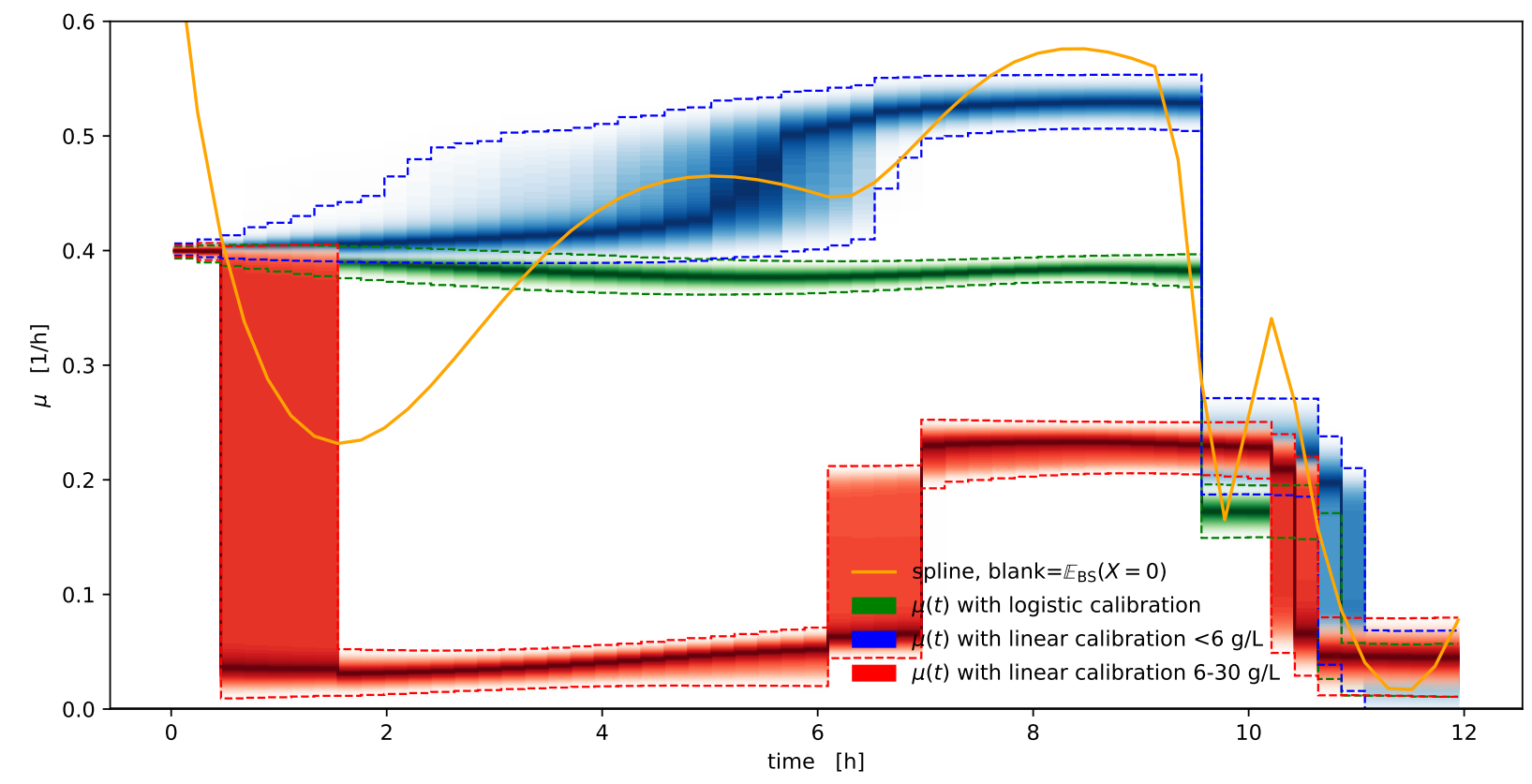

Figure 9: Comparison of growth rate calculation methods

Spline-based $\mu(t)$ growth rate calculation based on blank subtraction (orange) yields a point estimate that fluctuates considerably compared to the "gold standard" of the generative $\vec{\mu}_{t}$ method with detailed biomass/backscatter calibration (green). When the generative method is used with linear calibration models, the choice of calibration concentrations and the decision for (blue) or against (red) fixing the intercept at a blank backscatter has considerable effects on the quality of the outcome. The density bands visualize the posterior probability density, with dashed lines marking the 5 and $95 \%$ percentiles.

\subsection{Time series feature extraction}

It was previously shown that high-resolution timeseries of culture backscatter can be correlated with product measurements through the use of dimension-reduction techniques and regression models [22]. With bletl.features we provide an implementation for configurable and automated extraction of large numbers of features from bioprocess timeseries data. These features may be used as the input to a broad spectrum of machine learning pipelines making use of techniques such as dimension reduction, regression, unsupervised visualization or clustering.

To demonstrate how one might use these methods, we applied them to the previously introduced dataset to obtain 
a visualization of local structures in the high-dimensional data. Initially 2343 time series features were extracted from the full dataset using both biologically motivated, as well as the statistical time series feature extractors and cleaned to a set of 1282 features (Section 2.2.3). For visualization of the dataset we applied t-distributed Stochastic Neighbor Embedding (t-SNE, Section 2.2.4) to find a 2-dimensional embedding that maintains the local structure of the 1282-dimensional data. The result is a 2-dimensional arrangement (Figure 10) such that culture wells with similar on-line signals are located in close proximity.

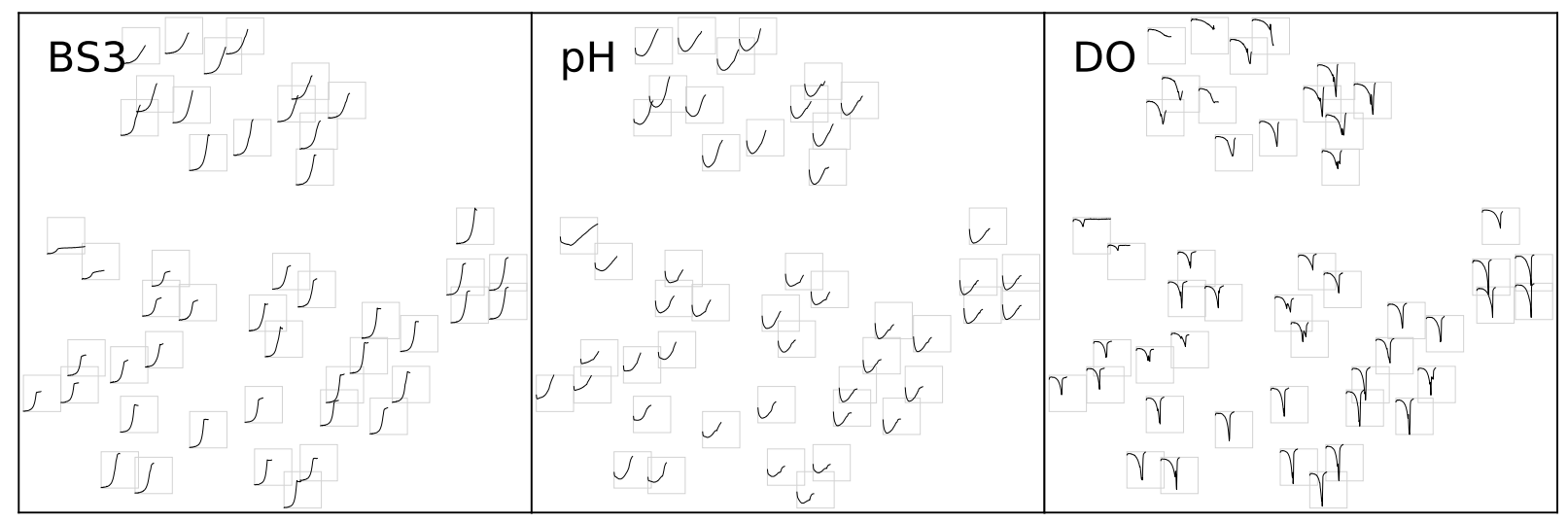

Figure 10: t-SNE from extracted time series features

Each small tile, arranged according to the t-SNE result, shows the time series for one well truncated at the time of harvest. Axis limits of the small tiles are identical within each of the three panels. As with any t-SNE visualization, the large-scale arrangement, rotation or axis units are meaningless, since the technique prioritizes local structure. Note that tiles arranged in close proximity are have similar time series characteristics in all three filtersets.

Coloring the embedding according to the carbon source composition (Figure 11) reveals that the arrangement found by t-SNE is strongly correlated with the presence of gluconate, glutamate and particularly lactate in the cultivation medium.

The observation that a t-SNE of extracted time series features does not only recover similarities between individual wells, but also aspects of the experiment design shows that our feature extraction is a viable solution to make BioLector datasets amendable to machine learning methods. In contrast to the extraction of manually engineered features [22], our feature extraction workflow works out of the box and with few lines of code. 


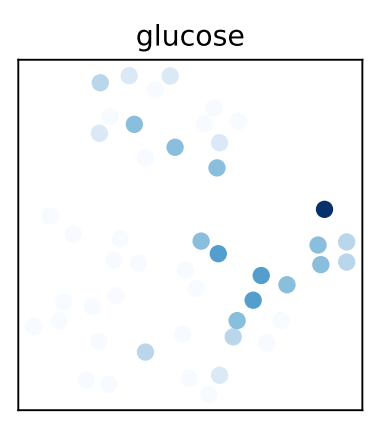

gluconat

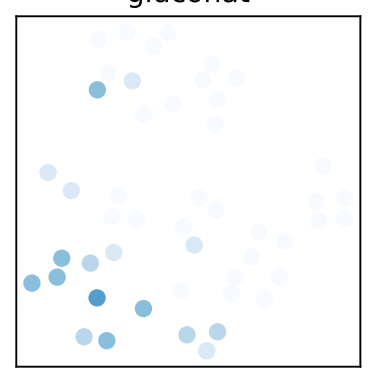

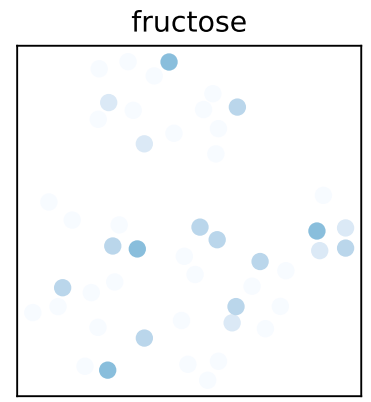

lactate

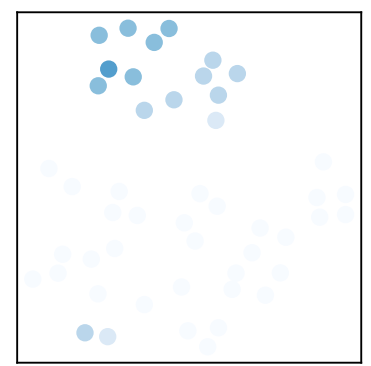

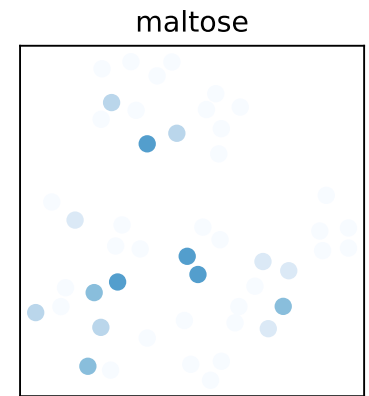

glutamate

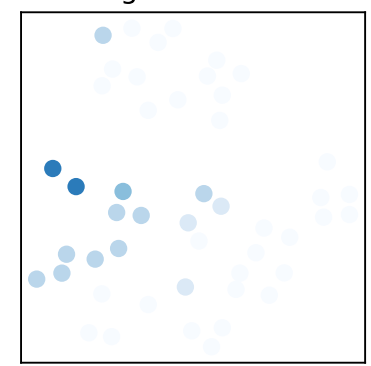

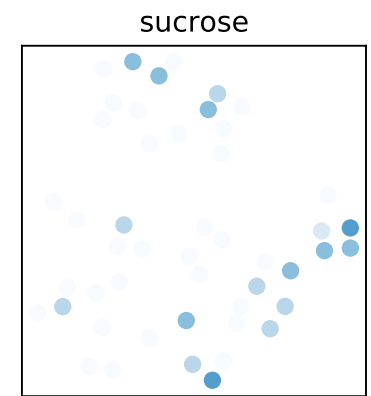

myo-inositol

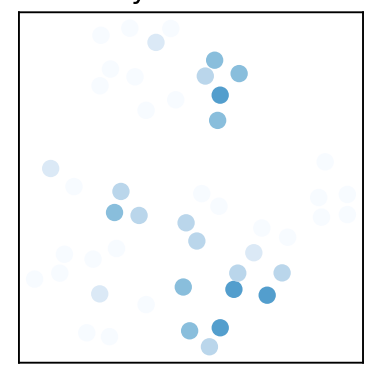

Figure 11: t-SNE embedding colored by carbon sources

Almost all wells that included lactate as a carbon source are closely arranged in the t-SNE embedding, indicating that they are in close proximity in the high dimensional feature space. Likewise, gluconate or glutamate containing wells are closely arranged. In contrast, none of the monomeric or dimeric sugars lead to characteristic BS/pH/DO phenotypes.

\section{Concluding remarks}

With the examples in Section 3.1 we showed how bletl makes BioLector datasets accessible to standard Python-based data analysis workflows. The switch to Python-based data processing facilitates not only interactive and robust data analysis, but also enables the application of machine learning techniques such as crossvalidated smoothing splines to BioLector datasets. Nevertheless, many scientists who are not yet proficient in Python-based data analysis workflows might be concerned with the initial complexity of the learning curve. That is one of the reasons why the documentation of the bletl package comes with ready-to-use examples. The code of the library is thoroughly tested in automated test pipelines to reduce the chance of unexpected failures.

In Section 3.2 we characterized two strategies for smoothing noisy on-line signals and showed that subtle differences in implementation can have substantial consequences on the results. This again highlights the need for standardized data structures, robust data analysis routines and thoroughly tested, open-sourced implementations that are distributed through versioned releases.

For the analysis of specific growth rate under not necessarily unlimited exponential growth conditions, we presented a random-walk based $\vec{\mu}_{t}$ model that can also detect switchpoints automatically. Within seconds our method determines time-variable growth rates by optimization and by leveraging state of the art probabilistic machine learning, it also quantifies Bayesian uncertainties. We showed on a synthetic dataset that the method is not only unbiased, but also offers the practitioner a tuning knob for the bias-variance tradeoff between narrow uncertainties and growth rate flexibility (Figure 7). In comparison with alternative approaches we found that while analyses with less exact, or even 
without calibration models may still find the same general trends, a quantitative statement about specific growth rate can only be made with accurate calibrations Figure S1.

With Section 3.4 we presented a generally applicable method to extract features for machine learning applications from time series data of microbioreactor experiments. By visualizing the high-dimensional time series features with t-SNE we showed that the features indeed have the information content needed to reconstruct patterns from the experimental design. The visualization of a high-dimensional BioLector dataset in a 2-dimensional arrangement that maintains local structure (Figure 10) is just one example of how our bletl package enriches the exploratory data analysis of microbioreactor experiments.

Overall we conclude that Python packages to parse experimental data into standardized data structures are a valuable asset for quantitative, qualitative and exploratory research.

\section{Acknowledgements}

The bletl package was developed by Michael Osthege and Niklas Tenhaef. The random-walk $\vec{\mu}_{t}$ method was devised and implemented by Michael Osthege. Feature extraction and crossvalidation routines were prototyped by Rebecca Zyla using a comprehensive data set provided by Johannes Hemmerich. The strain and experimental workflow for the dataset used in this study were produced by Carolin Müller. Marco Oldiges, Stephan Noack and Wolfgang Wiechert reviewed the manuscript, organized funding and were responsible for supervision and project coordination. This work was funded by the German Federal Ministry of Education and Research (BMBF, Grant. No. 031B0463A) as part of the project "Digitalization In Industrial Biotechnology", DigInBio. The authors have declared no conflict of interest.

\section{References}

[1] Jens Nielsen and Jay D Keasling. "Engineering cellular metabolism”. In: Cell 164.6 (2016), pp. 1185-1197.

[2] Peter Rohe, Deepak Venkanna, Britta Kleine, Roland Freudl, and Marco Oldiges. "An automated workflow for enhancing microbial bioprocess optimization on a novel microbioreactor platform”. In: Microbial Cell Factories 11.1 (2012), pp. 1-14.

[3] Johannes Hemmerich, Stephan Noack, Wolfgang Wiechert, and Marco Oldiges. "Microbioreactor systems for accelerated bioprocess development". In: Biotechnology journal 13.4 (2018), p. 1700141.

[4] Beate Vieth, Swati Parekh, Christoph Ziegenhain, Wolfgang Enard, and Ines Hellmann. "A systematic evaluation of single cell RNA-seq analysis pipelines". In: Nature communications 10.1 (2019), pp. 1-11.

[5] Johannes Hemmerich, Wolfgang Wiechert, and Marco Oldiges. "Automated growth rate determination in high-throughput microbioreactor systems”. In: BMC research notes 10.1 (2017), pp. 1-7.

[6] MN Cruz Bournazou, T Barz, DB Nickel, DC Lopez Cárdenas, F Glauche, A Knepper, and P Neubauer. "Online optimal experimental re-design in robotic parallel fed-batch cultivation facilities". In: Biotechnology and bioengineering 114.3 (2017), pp. 610-619. 
[7] Roman Jansen, Niklas Tenhaef, Matthias Moch, Wolfgang Wiechert, Stephan Noack, and Marco Oldiges. "FeedER: a feedback-regulated enzyme-based slow-release system for fed-batch cultivation in microtiter plates". In: Bioprocess and biosystems engineering 42.11 (2019), pp. 1843-1852.

[8] Wes McKinney. "Data Structures for Statistical Computing in Python". In: Proceedings of the 9th Python in Science Conference. Ed. by Stéfan van der Walt and Jarrod Millman. 2010, pp. 56-61. DOI: 10 .25080/Majora92bf 1922-00a.

[9] The pandas development team. pandas-dev/pandas: Pandas. Version latest. Feb. 2020. DOI: 10.5281/zenodo. 3509134. URL: https://doi.org/10.5281/zenodo.3509134.

[10] Pauli Virtanen et al. "SciPy 1.0: Fundamental Algorithms for Scientific Computing in Python". In: Nature Methods 17 (2020), pp. 261-272. DOI: 10.1038/s41592-019-0686-2.

[11] Eugene Prilepin. CSAPS - Cubic Spline Approximation (Smoothing). Version 1.0.4. June 8, 2021. URL: https: //github.com/espdev/csaps.

[12] Michael Osthege, Niklas Tenhaef, and Laura Helleckes. JuBiotech/bletl: v1.0.0. Version v1.0.0. July 2021. DOI: 10.5281/zenodo.5101435. URL: https://doi.org/10.5281/zenodo. 5101435.

[13] John Salvatier, Thomas V. Wiecki, and Christopher Fonnesbeck. "Probabilistic programming in Python using PyMC3”. In: PeerJ Computer Science 2 (Apr. 2016), e55. DOI: 10 . 7717 / peerj-cs . 55. URL: https : //doi.org/10.7717/peerj-cs.55.

[14] John Salvatier et al. pymc-devs/pymc3: PyMC3 3.11.2 (14 March 2021). Version v3.11.2. Mar. 2021. DOI: 10.5281/zenodo.4603971. URL: https://doi.org/10.5281/zenodo. 4603971.

[15] H. D. K. Moonesinghe and Pang-Ning Tan. "OutRank: A Graph-based outlier detection framework using random walk”. In: International Journal on Artificial Intelligence Tools 17.01 (2008), pp. 19-36. DOI: 10 . 1142 / S0218213008003753. eprint: https : / / doi . org/10 . 1142/S0218213008003753. URL: https: //doi.org/10.1142/S0218213008003753.

[16] Maximilian Christ, Nils Braun, Julius Neuffer, and Andreas W. Kempa-Liehr. "Time Series FeatuRe Extraction on basis of Scalable Hypothesis tests (tsfresh - A Python package)”. In: Neurocomputing 307 (2018), pp. $72-77$. ISSN: 0925-2312. DOI: https : / / doi .org/10 . 1016/j . neucom . 2018.03 .067. URL: https : / /www . sciencedirect.com/science/article/pii/S0925231218304843.

[17] bletl Documentation. URL: https://bletl.readthedocs.io.

[18] Patrick J. Bakkes, Paul Ramp, Astrid Bida, Doris Dohmen-Olma, Michael Bott, and Roland Freudl. "Improved pEKEx2-derived expression vectors for tightly controlled production of recombinant proteins in Corynebacterium glutamicum". In: Plasmid 112 (Nov. 2020), p. 102540. DOI: 10.1016/j ·plasmid. 2020 . 102540.

[19] Laura M. Helleckes, Michael Osthege, Wolfgang Wiechert, Eric von Lieres, and Marco Oldiges. "Bayesian calibration, process modeling and uncertainty quantification in biotechnology”. In: bioRxiv (2021). DOI: 10 . $1101 / 2021.06 .30 .450546$. 
bletl - A Python package for integrating microbioreactors in the Design-Build-Test-Learn cycle

[20] Carolin Müller, Chika L Igwe, Wolfgang Wiechert, and Marco Oldiges. "Scaling production of GFP1-10 detector protein in E. coli for secretion screening by split GFP assay". In: Microbial Cell Factories (2021 (minor revision)).

[21] Johannes Hemmerich, Niklas Tenhaef, Wolfgang Wiechert, and Stephan Noack. "pyFOOMB: Python framework for object oriented modeling of bioprocesses". In: Engineering in Life Sciences 21.3-4 (2021), pp. 242-257. DOI: https://doi.org/10.1002/elsc.202000088. URL: https://onlinelibrary.wiley.com/doi/abs/ 10.1002 /elsc. 202000088.

[22] Tobias Ladner, Martina Mühlmann, Andreas Schulte, Georg Wandrey, and Jochen Büchs. "Prediction of Escherichia coli expression performance in microtiter plates by analyzing only the temporal development of scattered light during culture”. In: Journal of biological engineering 11.1 (2017), pp. 1-15.

[23] Michael Osthege. JuBiotech/bletl-paper: v1.0.0. Version v1.0.0. Aug. 2021. DOI: 10.5281/zenodo. 5235461. URL: https://doi.org/10.5281/zenodo.5235461. 
bletl - A Python package for integrating microbioreactors in the Design-Build-Test-Learn cycle

\section{Appendix}

\subsection{Datasets used in this study}

The following dataset files can be found in the data directory of the supporting information GitHub repository [23]:

- 8X4PF4.csv is the raw BioLector dataset.

-8X4PF4_eventlog.Xlsx contains metadata of induction and sampling events.

- 8X4PF4_medium_composition.Xlsx is the well-wise composition of carbon sources in the growth media.

\subsection{Extracted features and t-SNE components}

The following files with results from the timeseries feature extraction and embedding are located in the results directory of the supporting information GitHub repository [23]:

- 8X4PF4_embedding. $\mathrm{xlsx}$ are the two t-SNE components that were used to arrange data points in the t-SNE visualizations.

- 8X4PF4_extracted_features contains the raw and cleaned well-wise features extracted from the BioLector dataset. 
bioRxiv preprint doi: https://doi.org/10.1101/2021.08.24.457462; this version posted August 25, 2021. The copyright holder for this preprint (which was not certified by peer review) is the author/funder, who has granted bioRxiv a license to display the preprint in perpetuity. It is made available under aCC-BY 4.0 International license.

bletl - A Python package for integrating microbioreactors in the Design-Build-Test-Learn cycle
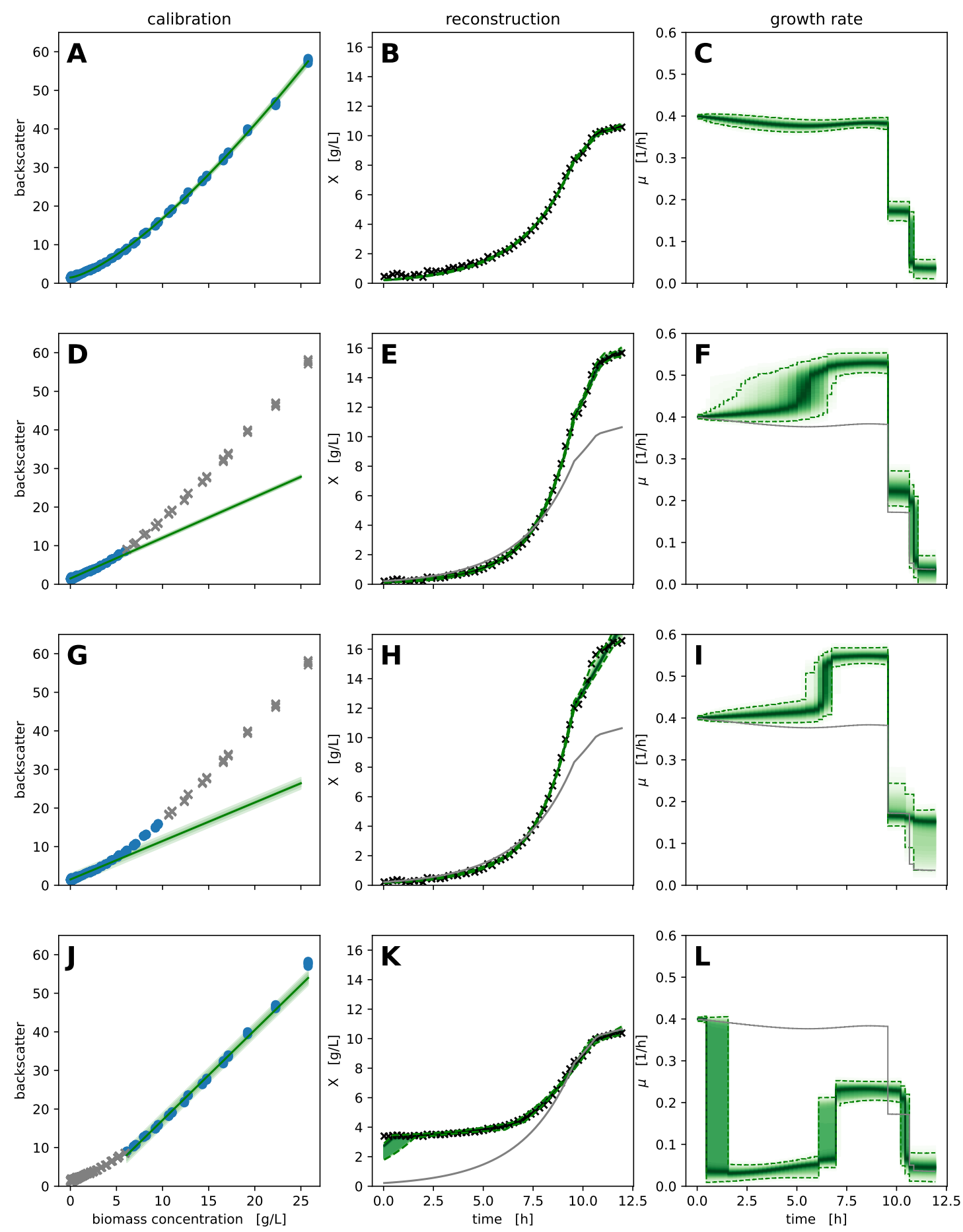

Figure S1: Influence of calibration models on the result of random-walk based $\vec{\mu}_{t}$ growth rates

An asymmetric logistic calibration model that accurately describes the calibration dataset (A) leads to a $\vec{\mu}_{t}$ profile (C) that is used as the baseline (grey) in the other plots. With a linear calibration model fitted only to calibration data up to $6 \mathrm{~g} / \mathrm{L}$ (D) or the expected maximum biomass concentration of $10 \mathrm{~g} / \mathrm{L}(\mathbf{G})$, the growth rate is systematically overestimated (F, I). A linear calibration model through only high biomass concentrations $(\mathbf{J})$ that would be amenable to individual cell dry weight determination (e.g. $>6 \mathrm{~g} / \mathrm{L}$ leads to an under-estimation of the growth rate, no longer follows the same profile and does not reliably detect switchpoints $(\mathbf{K}, \mathbf{L})$. The green density bands of the calibration models (left column) are likelihood bands showing the expected spread of observations, whereas the density bands in the middle and right column show the posterior probability density, with dashed lines marking the 5 and $95 \%$ percentiles. 


\subsection{Plasmid sequence of pCMEx8-NprE-Cutinase}

5'-GCACCAATGCTTCTGGCGTCAGGCAGCCATCGGAAGCTGTGGTATGGCTGTGCAGGTCGTAAATCACTGCATAATTCGTGTCGCTCAAGGCGCACTCCCGTTCTGGATAATGT TTTTTGCGCCGACATCATAACGGTTCTGGCAAATATTCTGAAATGAGCTGTTGACAATTAATCATCGGCTCGTATAATGTGTGGAATTGTGAGCGGATAACAATTTCACACAGCA AACAGAATTAAAAGATAGGACCAGGATTACGCCAAGCTTGCAGGCCTGCAGAGAAGACAGGAGAAAAACATATGGGTTTAGGTAAGAAATTGTCTGTTGCTGTCGCTGCTTCG TTTATGAGTTTATCAATCAGCCTGCCAGGTGTTCAGGCTGAATTCCCTACTAGTAACCCTGCTCAGGAGCTTGAGGCGCGCCAGCTTGGTAGAACAACTCGCGACGATCTGATCA ACGGCAATAGCGCTTCCTGCGCCGATGTCATCTTCATTTATGCCCGGGGTTCAACAGAGACGGGCAACTTGGGAACTCTCGGTCCTAGCATTGCCTCCAACCTTGAGTCCGCCTT CGGCAAGGACGGTGTCTGGATTCAGGGCGTTGGCGGTGCCTACCGAGCCACTCTTGGAGACAATGCTCTCCCTCGCGGTACCTCTAGCGCCGCAATCAGGGAGATGCTTGGCCT CTTCCAGCAGGCCAACACCAAGTGCCCCGACGCGACTTTGATCGCCGGTGGCTACAGCCAGGGTGCTGCACTTGCGGCCGCCTCCATCGAGGACCTCGACTCGGCCATTCGTGA CAAGATCGCCGGAACTGTTCTGTTCGGCTACACCAAGAACCTACAGAACCGTGGCCGAATCCCCAACTACCCTGCCGACAGGACCAAGGTGTTCTGCAATACAGGAGATCTCGT TTGTACTGGTAGCTTGATCGTTGCTGCACCTCACTTGGCTTATGGTCCTGATGCTCGGGGCCCTGCCCCTGAGTTCCTCATCGAGAAGGTTCGGGCTGTCCGTGGTTCTGCTCTTA TCGGCTCTGATGGCGGCTCTGGCGGCGGCTCTACATCTCGTGATCACATGGTTCTTCATGAATACGTTAACGCTGCTGGCATCACATAAGAGCTCAATACACTGGCCGTCTTCGTT TTACAGCCAAGCTTGGCTGTTTTGGCGGATGAGAGAAGATTTTCAGCCTGATACAGATTAAATCAGAACGCAGAAGCGGTCTGATAAAACAGAATTTGCCTGGCGGCAGTAGCG CGGTGGTCCCACCTGACCCCATGCCGAACTCAGAAGTGAAACGCCGTAGCGCCGATGGTAGTGTGGGGTCACCCCATGCGAGAGTAGGGAACTGCCAGGCATCAAATAAAACG AAAGGCTCAGTCGAAAGACTGGGCCTTTCGTTTTATCTGTTGTTTGTCGGTGAACGCTCTCCTGAGTAGGACAAATCCGCCGGGAGCGGATTTGAACGTTGCGAAGCAACGGCCC GGAGGGTGGCGGGCAGGACGCCCGCCATAAACTGCCAGGCATCAAATTAAGCAGAAGGCCATCCTGACGGATGGCCTTTTTGCGTTTCTACAAACTCTTTTGTTTATTTTTCTAA ATACATTCAAATATGTATCCGCTCATGAGACAATAACCCTGATAAATGCTTCAATAATATTGAAAAAGGAAGAGTATGAGTATTCAACATTTCCGTGTCGCCCTTATTCCCTTTTT TGCGGCATTTTGCCTTCCTGTTTTTGCTCACCCAGAAACGCTGGTGAAAGTAAAAGATGCTGAAGATCAGTTGGGTGCACGAGTGGGTTACATCGAACTGGATCTCAACAGCGGT AAGATCCTTGAGAGTTTTCGCCCCGAAGAACGTTTTCCAATGATGAGCACTTTTAAAGTTCTGCTATGTGGCGCGGTATTATCCCGTGTTGACGCCGGGCAAGAGCAACTCGGTC GCCGCATACACTATTCTCAGAATGACTTGGTTGAGTAATTCGTAATCATGTCATAGGCTCTTCCGCTTCCTCGCTCACTGACTCGCTGCGCTCGGTCGTTCGGCTGCGGCGAGCGG TATCAGCTCACTCAAAGGCGGTAATACGGTTATCCACAGAATCAGGGGATAACGCAGGAAAGAACATGTGAGCAAAAGGCCAGCAAAAGGCCAGGAACCGTAAAAAGGCCGC GTTGCTGGCGTTTTTCCATAGGCTCCGCCCCCCTGACGAGCATCACAAAAATCGACGCTCAAGTCAGAGGTGGCGAAACCCGACAGGACTATAAAGATACCAGGCGTTTCCCCC TGGAAGCTCCCTCGTGCGCTCTCCTGTTCCGACCCTGCCGCTTACCGGATACCTGTCCGCCTTTCTCCCTTCGGGAAGCGTGGCGCTTTCTCATAGCTCACGCTGTAGGTATCTCA GTTCGGTGTAGGTCGTTCGCTCCAAGCTGGGCTGTGTGCACGAACCCCCCGTTCAGCCCGACCGCTGCGCCTTATCCGGTAACTATCGTCTTGAGTCCAACCCGGTAAGACACGA CTTATCGCCACTGGCAGCAGCCACTGGTAACAGGATTAGCAGAGCGAGGTATGTAGGCGGTGCTACAGAGTTCTTGAAGTGGTGGCCTAACTACGGCTACACTAGAAGAACAGT ATTTGGTATCTGCGCTCTGCTGAAGCCAGTTACCTTCGGAAAAAGAGTTGGTAGCTCTTGATCCGGCAAACAAACCACCGCTGGTAGCGGTGGTTTTTTTGTTTGCAAGCAGCAG ATTACGCGCAGAAAAAAAGGATCTCAAGAAGATCCTTTGATCTTTTCTACGGGGTCTGACGCTCAGTGGAACGAAAACTCACGTTAAGGGATTTTGGTCATGAGATTATCAAAA AGGATCTTCACCTAGATCCTTTTGGGGGGGGGGGAAAGCCACGTTGTGTCTCAAAATCTCTGATGTTACATTGCACAAGATAAAAATATATCATCATGAACAATAAAACTGTCT GCTTACATAAACAGTAATACAAGGGGTGTTATGAGCCATATTCAACGGGAAACGTCTTGCTCGAGGCCGCGATTAAATTCCAACATGGATGCTGATTTATATGGGTATAAATGG GCTCGCGATAATGTCGGGCAATCAGGTGCGACAATCTATCGATTGTATGGGAAGCCCGATGCGCCAGAGTTGTTTCTGAAACATGGCAAAGGTAGCGTTGCCAATGATGTTACA GATGAGATGGTCAGACTAAACTGGCTGACGGAATTTATGCCTCTTCCGACCATCAAGCATTTTATCCGTACTCCTGATGATGCATGGTTACTCACCACTGCGATCCCCGGGAAAA CAGCATTCCAGGTATTAGAAGAATATCCTGATTCAGGTGAAAATATTGTTGATGCGCTGGCAGTGTTCCTGCGCCGGTTGCATTCGATTCCTGTTTGTAATTGTCCTTTTAACAGC GATCGCGTATTTCGTCTCGCTCAGGCGCAATCACGAATGAATAACGGTTTGGTTGATGCGAGTGATTTTGATGACGAGCGTAATGGCTGGCCTGTTGAACAAGTCTGGAAAGAA ATGCATAAGCTTTTGCCATTCTCACCGGATTCAGTCGTCACTCATGGTGATTTCTCACTTGATAACCTTATTTTTGACGAGGGGAAATTAATAGGTTGTATTGATGTTGGACGAGT CGGAATCGCAGACCGATACCAGGATCTTGCCATCCTATGGAACTGCCTCGGTGAGTTTTCTCCTTCATTACAGAAACGGCTTTTTCAAAAATATGGTATTGATAATCCTGATATG AATAAATTGCAGTTTCATTTGATGCTCGATGAGTTTTTCTAATCAGAATTGGTTAATTGGTTGTAACACTGGCAGAGCATTACGCTGACTTGACGGGACGGCGGCTTTGTTGAAT AAATCGAACTTTTGCTGAGTTGAAGGATCAGATCACGCATCTTCCCGACAACGCAGACCGTTCCGTGGCAAAGCAAAAGTTCAAAATCACCAACTGGTCCACCTACAACAAAGC TCTCATCAACCGTGGCTCCCTCACTTTCTGGCTGGATGATGGGGCGATTCAGGCCTGGTATGAGTCAGCAACACCTTCTTCACGAGGCAGACCTCAGCGCCCCCCCCCCCCTAGC TTGTCTACGTCTGATGCTTTGAATCGGACGGACTTGCCGATCTTGTATGCGGTGATTTTTCCCTCGTTTGCCCACTTTTTAATGGTGGCCGGGGTGAGAGCTACGCGGGCGGCGAC CTGCTGCGCTGTGATCCAATATTCGGGGTCGTTCACTGGTTCCCCTTTCTGATTTCTGGCATAGAAGAACCCCCGTGAACTGTGTGGTTCCGGGGGTTGCTGATTTTTGCGAGACT TCTCGCGCAATTCCCTAGCTTAGGTGAAAACACCATGAAACACTAGGGAAACACCCATGAAACACCCATTAGGGCAGTAGGGCGGCTTCTTCGTCTAGGGCTTGCATTTGGGCG GTGATCTGGTCTTTAGCGTGTGAAAGTGTGTCGTAGGTGGCGTGCTCAATGCACTCGAACGTCACGTCATTTACCGGGTCACGGTGGGCAAAGAGAACTAGTGGGTTAGACATT GTTTTCCTCGTTGTCGGTGGTGGTGAGCTTTTCTAGCCGCTCGGTAAACGCGGCGATCATGAACTCTTGGAGGTTTTCACCGTTCTGCATGCCTGCGCGCTTCATGTCCTCACGTA GTGCCAAAGGAACGCGTGCGGTGACCACGACGGGCTTAGCCTTTGCCTGCGCTTCTAGTGCTTCGATGGTGGCTTGTGCCTGCGCTTGCTGCGCCTGTAGTGCCTGTTGAGCTTC TTGTAGTTGCTGTTCTAGCTGTGCCTTGGTTGCCATGCTTTAAGACTCTAGTAGCTTTCCTGCGATATGTCATGCGCATGCGTAGCAAACATTGTCCTGCAACTCATTCATTATGTG CAGTGCTCCTGTTACTAGTCGTACATACTCATATTTACCTAGTCTGCATGCAGTGCATGCACATGCAGTCATGTCGTGCTAATGTGTAAAACATGTACATGCAGATTGCTGGGGG TGCAGGGGGCGGAGCCACCCTGTCCATGCGGGGTGTGGGGCTTGCCCCGCCGGTACAGACAGTGAGCACCGGGGCACCTAGTCGCGGATACCCCCCTAGGTATCGGACACGT AACCCTCCCATGTCGATGCAAATCTTTAACATTGAGTACGGGTAAGCTGGCACGCATAGCCAAGCTAGGCGGCCACCAAACACCACTAAAAATTAATAGTTCCTAGACAAGACA AACCCCCGTGCGAGCTACCAACTCATATATGCACGGGGGCCACATAACCCGAAGGGGTTTCAATTGACAACCATAGCACTAGCTAAGACAACGGGCACAACACCCGCACAAAC TCGCACTGCGCAACCCCGCACAACATCGGGTCTAGGTAACACTGAAATAGAAGTGAACACCTCTAAGGAACCGCAGGTCAATGAGGGTTCTAAGGTCACTCGCGCTAGGGCGT GGCGTAGGCAAAACGTCATGTACAAGATCACCAATAGTAAGGCTCTGGCGGGGTGCCATAGGTGGCGCAGGGACGAAGCTGTTGCGGTGTCCTGGTCGTCTAACGGTGCTTCGC AGTTTGAGGGTCTGCAAAACTCTCACTCTCGCTGGGGGTCACCTCTGGCTGAATTGGAAGTCATGGGCGAACGCCGCATTGAGCTGGCTATTGCTACTAAGAATCACTTGGCGGC GGGTGGCGCGCTCATGATGTTTGTGGGCACTGTTCGACACAACCGCTCACAGTCATTTGCGCAGGTTGAAGCGGGTATTAAGACTGCGTACTCTTCGATGGTGAAAACATCTCAG TGGAAGAAAGAACGTGCACGGTACGGGGTGGAGCACACCTATAGTGACTATGAGGTCACAGACTCTTGGGCGAACGGTTGGCACTTGCACCGCAACATGCTGTTGTTCTTGGAT CGTCCACTGTCTGACGATGAACTCAAGGCGTTTGAGGATTCCATGTTTTCCCGCTGGTCTGCTGGTGTGGTTAAGGCCGGTATGGACGCGCCACTGCGTGAGCACGGGGTCAAAC TTGATCAGGTGTCTACCTGGGGTGGAGACGCTGCGAAAATGGCAACCTACCTCGCTAAGGGCATGTCTCAGGAACTGACTGGCTCCGCTACTAAAACCGCGTCTAAGGGGTCGT 
ACACGCCGTTTCAGATGTTGGATATGTTGGCCGATCAAAGCGACGCCGGCGAGGATATGGACGCTGTTTTGGTGGCTCGGTGGCGTGAGTATGAGGTTGGTTCTAAAAACCTGC GTTCGTCCTGGTCACGTGGGGCTAAGCGTGCTTTGGGCATTGATTACATAGACGCTGATGTACGTCGTGAAATGGAAGAAGAACTGTACAAGCTCGCCGGTCTGGAAGCACCGG AACGGGTCGAATCAACCCGCGTTGCTGTTGCTTTGGTGAAGCCCGATGATTGGAAACTGATTCAGTCTGATTTCGCGGTTAGGCAGTACGTTCTAGATTGCGTGGATAAGGCTAA GGACGTGGCCGCTGCGCAACGTGTCGCTAATGAGGTGCTGGCAAGTCTGGGTGTGGATTCCACCCCGTGCATGATCGTTATGGATGATGTGGACTTGGACGCGGTTCTGCCTACT CATGGGGACGCTACTAAGCGTGATCTGAATGCGGCGGTGTTCGCGGGTAATGAGCAGACTATTCTTCGCACCCACTAAAAGCGGCATAAACCCCGTTCGATATTTTGTGCGATG AATTTATGGTCAATGTCGCGGGGGCAAACTATGATGGGTCTTGTTGTTGACAATGGCTGATTTCATCAGGAATGGAACTGTCATGCTGTTATGTGCCTGGCTCCTAATCAAAGCT GGGGACAATGGGTTGCCCCGTTGATCTGATCTAGTTCGGATTGGCGGGGCTTCACTGTATCTGGGGGTGGCATCGTGAATAGATTGCACACCGTAGTGGGCAGTGTGCACACCA TAGTGGCCATGAGCACCACCACCCCCAGGGACGCCGACGGCGCGAAGCTCTGCGCCTGGTGCGGCTCGGAGATCAAGCAATCCGGCGTCGGCCGGAGCCGGGACTACTGCCGC CGCTCCTGCCGCCAGCGGGCGTACGAGGCCCGGCGCCAGCGCGAGGCGATCGTGTCCGCCGTGGCGTCGGCAGTCGCTCGCCGAGATACGTCACGTGACGAAATGCAGCAGCC TTCCATTCCGTCACGTGACGAAACTCGGGCCGCAGGTCAGAGCACGGTTCCGCCCGCTCCGGCCCTGCCGGACCCCCGGCATCCCGCAAGAGGCCCGGCAGTACCGGCATAACC AAGCCTATGCCTACAGCATCCAGGGTGACGGTGCCGAGGATGACGATGAGCGCATTGTTAGATTTCATACACGGTGCCTGACTGCGTTAGCAATTTAACTGTGATAAACTACCG CATTAAAGCTTATCGATGATAAGCTGTCAAACATGGCCTGTCGCTTGCGGTATTCGGAATCTTGCACGCCCTCGCTCACTGCCCGCTTTCCAGTCGGGAAACCTGTCGTGCCAGC TGCATTAATGAATCGGCCAACGCGCGGGGAGAGGCGGTTTGCGTATTGGGCGCCAGGGTGGTTTTTCTTTTCACCAGTGAGACGGGCAACAGCTGATTGCCCTTCACCGCCTGG CCCTGAGAGAGTTGCAGCAAGCGGTCCACGCTGGTTTGCCCCAGCAGGCGAAAATCCTGTTTGATGGTGGTTAACGGCGGGATATAACATGAGCTGTCCTCGGTATCGTCGTAT CCCACTACCGAGATATCCGCACCAACGCGCAGCCCGGACTCGGTAATGGCGCGCATTGCGCCCAGCGCCATCTGATCGTTGGCAACCAGCATCGCAGTGGGAACGATGCCCTCA TTCAGCATTTGCATGGTTTGTTGAAAACCGGACATGGCACTCCAGTCGCCTTCCCGTTCCGCTATCGGCTGAATTTGATTGCGAGTGAGATATTTATGCCAGCCAGCCAGACGCA GACGCGCCGAGACAGAACTTAATGGGCCCGCTAACAGCGCGATTTGCTGGTGACCCAATGCGACCAGATGCTCCACGCCCAGTCGCGTACCGTCCTCATGGGAGAAAATAATAC TGTTGATGGGTGTCTGGTCAGAGACATCAAGAAATAACGCCGGAACATTAGTGCAGGCAGCTTCCACAGCAATGGCATCCTGGTCATCCAGCGGATAGTTAATGATCAGCCCAC TGACGCGTTGCGCGAGAAGATTGTGCACCGCCGCTTTACAGGCTTCGACGCCGCTTCGTTCTACCATCGACACCACCACGCTGGCACCCAGTTGATCGGCGCGAGATTTAATCGC CGCGACAATTTGCGACGGCGCGTGCAGGGCCAGACTGGAGGTGGCAACGCCAATCAGCAACGACTGTTTGCCCGCCAGTTGTTGTGCCACGCGGTTGGGAATGTAATTCAGCTC CGCCATCGCCGCTTCCACTTTTTCCCGCGTTTTCGCAGAAACGTGGCTGGCCTGGTTCACCACGCGGGAAACGGTCTGATAAGAGACACCGGCATACTCTGCGACATCGTATAAC GTTACTGGTTTCACATTCACCACCCTGAATTGACTCTCTTCCGGGCGCTATCATGCCATACCGCGAAAGGTTTTGCACCATTCGATGGTGTCAACGTAAATGCATGCCGCTTCGCC TTCGCGCGCGAATTGCAAGCTGATCCGGGCTTATCGACTGCACGGT-3' 